\title{
THE HALTING PROBLEM RELATIVIZED TO COMPLEMENTS
}

\author{
LOUISE HAY ${ }^{1}$
}

\begin{abstract}
Let $H^{A}=\{e \mid$ domain $\{e\} \cap A \neq \varnothing\}$. It is shown that there exists a set $A$ of Turing degree $a$ such that $H^{A}$ is Turingincomparable to $H^{\bar{A}}$ whenever $a$ is an r.e. degree with $a^{\prime}>0^{\prime}$, or $a \geqq 0^{\prime \prime}$ or $a \geqq 0^{\prime}$ and $a$ is r.e. in $0^{\prime}$. This contrasts with the fact that $H^{\bar{A}}$ is comparable to $H^{\bar{A}}$ for almost all $A$.
\end{abstract}

1. Introduction. In [7], the "relativized halting problem" is defined to be the problem of deciding, for a fixed set $A$, whether domain $\{e\} \cap$ $A \neq \varnothing$, where $\{e\}$ denotes the $e$ th partial recursive function. It is shown in that paper that the difficulty of the halting problem relativized to $A$ is not a simple function of the "complexity" of $A$, in the sense that the Turing degree of $H^{A}=\{e \mid$ domain $\{e\} \cap A \neq \varnothing\}$ is, in general, independent of the Turing degree of $A$; the same result was obtained independently in [2] for the special case where $A$ is r.e., nonrecursive. (The context of [2] was not given as a "halting problem", but as the classification of $\theta A^{B}=\left\{e \mid W_{e} \subset B\right\}$ where $W_{e}=$ domain $\{e\}$; but evidently $\theta A^{B}=\bar{H}^{B}$, where $\bar{B}$ denotes the complement of $B$.) In both [2] and [7] it is shown that it is possible for $H^{A}$ and $H^{\bar{A}}$ to have different Turing degrees, and in [7] the author conjectures that there exists a set $A$ such that $H^{A}$ and $H^{\bar{A}}$ have incomparable Turing degrees. The purpose of this paper is to prove this conjecture true; such sets occur, in fact, in all Turing degrees $a$ satisfying one of the following conditions:

(i) $\boldsymbol{a}$ is an r.e. degree with $\boldsymbol{a}^{\prime}>\mathbf{0}^{\prime}$;

(ii) $\boldsymbol{a} \geqq \mathbf{0}^{\prime \prime}$;

(iii) $\boldsymbol{a} \geqq 0^{\prime}$ and $\boldsymbol{a}$ is r.e. in $\mathbf{0}^{\prime}$.

In the concluding section we discuss why some such restrictions are necessary.

2. Main results. The notation is that of [5], except that $\{2 x \mid x \in A\} \cup$ $\{2 x+1 \mid x \in B\}$ will be denoted by $A \oplus B$. The Turing degree of $A$ will be denoted by $d(A)$; in this notation, $d(A \oplus B)=d(A) \cup d(B)$. Following

Received by the editors February 26, 1973.

AMS (MOS) subject classifications (1970). Primary 02F30, $02 \mathrm{~F} 25$.

${ }^{1}$ This research was supported by a sabbatical from the University of Illinois at Chicago Circle. I would like to thank the Mathematics Department at Rutgers University for its hospitality.

(c) American Mahtematical Society 1973 
[3], a set $A$ is called bi-immune if $A$ and $\bar{A}$ are immune, and a degree is called bi-immune if it contains a bi-immune set. A set $A$ is called d.r.e. if there exist r.e. sets $B$ and $C$ with $A=B-C$. For any set $A, \tilde{A}$ will denote $\left\{\langle u, v\rangle \mid D_{u} \subset A \& D_{v} \subset \bar{A}\right\}$, where $\left\{D_{i}\right\}$ is the canonical indexing of finite sets. The techniques used in our proofs are based on those of [7], but some additional work is needed to control complements.

THEOREM 1. Let a be an r.e. degree with $\boldsymbol{a}^{\prime}>0^{\prime}$. Then there exists a set $A$ satisfying

(i) $d(A)=\boldsymbol{a}$,

(ii) $d\left(H^{A}\right) \mid d\left(H^{\bar{A}}\right)$,

(iii) $A$ and $\bar{A}$ are d.r.e.

We first list some known results which will be needed for the proof.

Fact 1 (Robinson [4]). If $\boldsymbol{a} \leqq \boldsymbol{b}<\boldsymbol{c}$ and $\boldsymbol{b}, \boldsymbol{c}$ are r.e. in $\boldsymbol{a}$, then there exist degrees $b_{0}, b_{1}$ which are r.e. in $a$ and such that $b<b_{0}, b_{1}<c$ and $b_{0} \mid b_{1}$.

Fact 2 (Hay [2]). If $a$ is r.e., nonrecursive, and $b$ is a degree r.e. in $a$ with $\boldsymbol{b} \geqq 0^{\prime}$, then there exists an r.e. set $A$ such that $d(A)=a$ and $d\left(H^{\bar{A}}\right)=b$.

Fact 3 (Selman [7]). For all sets $A, B, d\left(H^{A \oplus B}\right)=d\left(H^{A}\right) \mathbf{U} d\left(H^{B}\right)$.

LeMmA 1. If $A$ is r.e. and nonempty, then $d\left(H^{A}\right)=0^{\prime}$.

Proof. If $A$ is r.e., $H^{A}$ is evidently also r.e., so $d\left(H^{A}\right) \leqq 0^{\prime}$. Conversely, $A \neq \varnothing$ implies $H^{A} \neq \varnothing$, so $0^{\prime} \leqq d\left(H^{A}\right)$ by Rice's theorem [5].

Lemma 2. If $A$ and $B$ are r.e., then $A \oplus \bar{B}$ and $(A \oplus \bar{B})^{-}$are d.r.e.

Proof. Let $E=\{x \mid x$ is even $\}, A_{0}=\{x \mid[x / 2] \in A\}, B_{0}=\{x \mid[x / 2] \in B\}$. Then $E, \bar{E}, A_{0}, B_{0}$ are clearly r.e., and $A \oplus \bar{B}=\left[\left(E \cap A_{0}\right) \cup \bar{E}\right]-B_{0}$, $(A \oplus \bar{B})^{-}=\bar{A} \oplus B=\left[E \cup\left(\bar{E} \cap B_{0}\right)\right]-A_{0}$.

Proof of Theorem 1. Suppose $a$ is r.e. and $a^{\prime}>0^{\prime}$. Then $a \leqq 0^{\prime}<a^{\prime}$, with $0^{\prime}$ and $\boldsymbol{a}^{\prime}$ both r.e. in $\boldsymbol{a}$. Hence by Fact 1 , there exist degrees $\boldsymbol{b}_{\mathbf{0}}, \boldsymbol{b}_{\mathbf{1}}$ r.e. in $a$ with $0^{\prime} \leqq b_{0}, b_{1}$ and $b_{0} \mid b_{1}$. By Fact 2 , there exist r.e. sets $A_{0}, A_{1}$ with $d\left(A_{0}\right)=d\left(A_{1}\right)=a, d\left(H^{\bar{A}_{0}}\right)=b_{0}$ and $d\left(H^{\bar{A}_{1}}\right)=\boldsymbol{b}_{1}$. Now $\boldsymbol{a}^{\prime}>\mathbf{0}^{\prime}$ implies $a>0$, hence $A_{0}$ and $A_{1}$ are nonempty; so by Lemma $1, d\left(H^{A_{0}}\right)=d\left(H^{A_{1}}\right)=0^{\prime}$. Let $A=A_{0} \oplus\left(A_{1}\right)^{-}$; clearly $d(A)=a$ and, by Fact $3, d\left(H^{A}\right)=d\left(H^{A_{0} \oplus A_{1}}\right)=$ $d\left(H^{A_{0}}\right) \cup d\left(H^{\bar{A}_{1}}\right)=\mathbf{0}^{\prime} \cup b_{1}=b_{1}$. Similarly, since $\bar{A}=\bar{A}_{0} \oplus A_{1}, d\left(H^{\bar{A}}\right)=d\left(H^{\bar{A}_{0}}\right) \cup$ $d\left(H^{A_{1}}\right)=b_{0} \cup 0^{\prime}=b_{0}$. So $d\left(H^{A}\right) \mid d\left(H^{\bar{A}}\right)$ and, by Lemma $2, A$ and $\bar{A}$ are d.r.e., which completes the proof.

Note that in Theorem 1, "d.r.e." cannot be improved to "r.e." even for one of the sets $A, \bar{A}$, since if $A$ is r.e. and neither $A$ nor $\bar{A}$ is empty, then $d\left(H^{A}\right)=0^{\prime} \leqq d\left(H^{\bar{A}}\right)$ by Lemma 1 and Rice's theorem.

THEOREM 2. Assume $0^{\prime} \leqq a \leqq b_{0}, b_{1}$. If there exist bi-immune degrees $c_{0}, c_{1}$ such that $c_{0}, c_{1} \leqq a, b_{0}=c_{0}^{\prime}$ and $b_{1}=c_{1}^{\prime}$, then there is a set $A$ satisfying $d(A)=a, d\left(H^{A}\right)=b_{0}, d\left(H^{\bar{A}}\right)=b_{1}$. 
We again list some known results needed for the proof.

Fact 4 (Selman [7]). Let $d(B)=b$. If $\bar{B}$ is immune, then $d\left(H^{B}\right)=b \mathbf{U} \mathbf{0}^{\prime}$.

Fact 5 [5, Exercise 9-31]. For all $a$, if $a \geqq 0^{\prime}$ then $a$ is bi-immune.

Fact 6 [5, Exercise 9-50]. If $\tilde{A}=\left\{\langle u, v\rangle \mid D_{u} \subset A \& D_{v} \subset \bar{A}\right\}$, then $d\left(H^{\tilde{A}}\right)=\boldsymbol{a}^{\prime}$.

Lemma 3. For all sets $A$, if $A$ is bi-immune then $\tilde{A}$ is immune.

Proof. Assume $A$ and $\bar{A}$ are both immune. In particular, they are then infinite, so they have infinitely many finite subsets and hence $\tilde{A}$ is infinite. Let $W$ be an r.e. subset of $\tilde{A}$. Define

$$
W_{0}=\{u \mid(\exists v)(\langle u, v\rangle \in W)\}, \quad W_{1}=\{v \mid(\exists u)(\langle u, v\rangle \in W)\} .
$$

Then $W_{0}$ and $W_{1}$ are r.e., and, for all $u, v, u \in W_{0}$ implies $D_{u} \subset A, v \in W_{1}$ implies $D_{v} \subset \bar{A}$. Suppose $W_{0}$ is infinite, and let

$$
V_{0}=\left\{x \mid(\exists u)\left(u \in W_{0} \& x \in D_{u}\right)\right\} .
$$

Then $V_{0}$ is infinite, since the canonical indexing is one-one and hence $V_{0}$ is the union of infinitely many different finite sets. But then $V_{0}$ is an infinite r.e. subset of $A$, which contradicts the assumption that $A$ is immune. So $W_{0}$ is finite, and by a symmetric argument using $W_{1}$ and $\bar{A}, W_{1}$ is also finite. But then $W_{0} \times W_{1}$ is finite, and hence so is $W$ since $W \subset W_{0} \times W_{1}$. So every r.e. subset of $\tilde{A}$ is finite, and $\tilde{A}$ is immune.

LeMma 4. Let a be bi-immune. Then there is a set $B$ such that $d(B)=a$, $d\left(H^{B}\right)=\boldsymbol{a}^{\prime}$ and $d\left(H^{\bar{B}}\right)=\boldsymbol{a} \mathbf{U} \mathbf{0}^{\prime}$.

Proof. Assume $a$ contains a bi-immune set $A$, and let $B=\tilde{A}$; clearly $d(B)=d(A)=a$. By Fact $6, d\left(H^{B}\right)=d\left(H^{\tilde{A}}\right)=\boldsymbol{a}^{\prime}$. By Lemma $3, B$ is immune, so $d\left(H^{\bar{B}}\right)=d(B) \cup 0^{\prime}=a \mathbf{U} 0^{\prime}$, by Fact 4 .

Proof of THeOReM 2. Assume $0^{\prime} \leqq a \leqq b_{0}, b_{1}$, and that there exist bi-immune degrees $c_{0}, c_{1}$ satisfying $c_{0}, c_{1} \leqq a, b_{0}=c_{0}^{\prime}, b_{1}=c_{1}^{\prime}$. By Lemma 4, there exist sets $C_{0}, C_{1}$ such that for $i=0,1, d\left(C_{i}\right)=c_{i}, d\left(H^{C_{i}}\right)=\boldsymbol{c}_{i}^{\prime}=\boldsymbol{b}_{i}$ and $d\left(H^{\bar{C}_{i}}\right)=c_{i} \cup 0^{\prime}$. By Fact $5,0^{\prime} \leqq a$ implies $\boldsymbol{a}$ is bi-immune. Let $A_{0}$ be any bi-immune set such that $d\left(A_{0}\right)=a$; then by Fact 4 ,

$$
d\left(H^{A_{0}}\right)=d\left(H^{\bar{A}_{0}}\right)=\boldsymbol{a} \cup \mathbf{0}^{\prime}=\boldsymbol{a} .
$$

Let $A=\left(C_{0} \oplus \bar{C}_{1}\right) \oplus A_{0}$; then $\bar{A}=\left(C_{0} \oplus \bar{C}_{1}\right)-\oplus \overline{A_{0}}=\left(\bar{C}_{0} \oplus C_{1}\right) \oplus \overline{A_{0}}$, and

$$
d(A)=d\left(C_{0}\right) \cup d\left(C_{1}\right) \cup d\left(A_{0}\right)=c_{0} \cup c_{1} \cup a=a
$$

since $c_{0}, c_{1} \leqq a$. By Fact 3 ,

$$
d\left(H^{\mathcal{A}}\right)=d\left(H^{C_{0}}\right) \cup d\left(H^{\bar{C}_{1}}\right) \cup d\left(H^{A_{0}}\right)=b_{0} \cup c_{1} \cup 0^{\prime} \cup a=b_{0}
$$

since $\boldsymbol{c}_{\mathbf{1}} \mathbf{\cup} \mathbf{0}^{\prime} \leqq \boldsymbol{a} \leqq \boldsymbol{b}_{\mathbf{0}}$, and, similarly,

$$
d\left(H^{\bar{A}}\right)=d\left(H^{\bar{C}_{0}}\right) \cup d\left(H^{C_{1}}\right) \cup d\left(H^{\bar{A}_{0}}\right)=c_{0} \cup 0^{\prime} \cup b_{1} \cup a=b_{1} .
$$

So $A$ satisfies the conclusion of the theorem. 
THEOREM 3. Let a be any degree such that $a \geqq 0^{\prime \prime}$. Then there exists a set $A$ such that $d(A)=a$ and $d\left(H^{A}\right) \mid d\left(H^{\bar{A}}\right)$.

The proof will be an application of Theorem 2. We list some additional facts which will be needed.

Fact 7 (Selman [6]). For all $a$, if $a \geqq 0^{\prime \prime}$ then there exists a degree $c$ such that $a=c^{\prime \prime}$.

Fact 8 (Shoenfield [8]). Let $b, c$ be degrees such that $c^{\prime} \leqq b$ and $b$ is r.e. in $c^{\prime}$. Then there is a degree $d$ with $c \leqq d \leqq c^{\prime}$ and $d^{\prime}=b$.

Proof of Theorem 3. Assume $a \geqq 0^{\prime \prime}$. By Fact $7, a=c^{\prime \prime}$ for some degree $c$. Apply Fact 1 to obtain degrees $b_{0}, b_{1}$ r.e. in $a$ with $a \leqq b_{0}, b_{1} \leqq a^{\prime}$ and $b_{0} \mid b_{1}$. (This special case of Fact 1 is of course just the FriedbergMuchnik theorem relativized to a.) Then by Fact 8, there exist degrees $c_{0}, c_{1}$ with $b_{0}=c_{0}^{\prime}, b_{1}=c_{1}^{\prime}$ and $0^{\prime} \leqq c^{\prime} \leqq c_{0}, c_{1} \leqq c^{\prime \prime}=a$. By Fact $5,0^{\prime} \leqq c_{0}, c_{1}$ implies $c_{0}$ and $c_{1}$ are bi-immune. The hypotheses of Theorem 2 therefore apply to $a, b_{0}, b_{1}$; hence there is a set $A$ such that $d(A)=a$ and $d\left(H^{A}\right)=$ $b_{0} \mid b_{1}=d\left(H^{A}\right)$.

THEOREM 4. If $a \geqq 0^{\prime}$ and $a$ is r.e. in $0^{\prime}$, then there exists a set $A$ such that $d(A)=a$ and $d\left(H^{\boldsymbol{A}}\right) \mid d\left(H^{\bar{A}}\right)$.

We list one more fact needed to apply Theorem 2.

Fact 9 (Jockusch [3]). If $\boldsymbol{d}$ has a nonzero predecessor $c$ which is r.e. in $\mathbf{0}^{\prime}$, then $\boldsymbol{d}$ is bi-immune.

Proof of Theorem 4. Assume $a \geqq 0^{\prime}$ and $a$ is r.e. in $0^{\prime}$. Then by Fact 8 , there exists a degree $c \leqq 0^{\prime}$ such that $a=c^{\prime}$. Again by 'Fact 1 , there exist degrees $b_{0}, b_{1}$ r.e. in $a$ with $a<b_{0}, b_{1}$ and $b_{0} \mid b_{1}$. Apply Fact 8 again to obtain degrees $c_{0}, c_{1}$ with $b_{0}=c_{0}^{\prime}, b_{1}=c_{1}^{\prime}$ and $c \leqq c_{0}, c_{1} \leqq c^{\prime}=a$. But $c \leqq 0^{\prime}$ implies $c$ is r.e. in $0^{\prime}$; hence by Fact $9, c_{0}, c_{1}$ are bi-immune. Thus the hypotheses of Theorem 2 again apply to $a, b_{0}, b_{1}$; hence there is a set $A$ satisfying $d(A)=a$ and $d\left(H^{A}\right)=b_{0} \mid b_{1}=d\left(H^{\bar{A}}\right)$.

3. Concluding remarks. To what extent are the conditions on $a$ in the hypotheses of Theorems 1, 3 and 4 necessary? In [7] it is pointed out that membership information concerning $H^{A}$ gives no information regarding membership in $H^{\bar{A}}$-and certainly in the case where $W_{e}$ is infinite it is not clear how one might hope to decide (in general) whether $W_{e} \cap \bar{A} \neq$ $\varnothing$ on the basis of finitely many questions of the type: Does $W_{x} \cap A=\varnothing$ ? It is tempting to conclude that $d\left(H^{A}\right) \mid d\left(H^{\tilde{A}}\right)$ must be the rule rather than the exception; but, somewhat curiously, this is not the case. For purposes of this discussion, call a set $A$ free if $d\left(H^{A}\right)$ is incomparable to $d\left(H^{\bar{A}}\right)$, bound otherwise. Call a degree $a$ totally free if every set in $\boldsymbol{a}$ is free, and 
totally bound if every set in $a$ is bound. The following then hold:

(a) Almost all sets $A$ are bound.

(b) No degree is totally free.

(c) Almost all degrees are totally bound.

Here "almost all" means "on a set of measure 1" in the usual measure on Baire space and the induced measure on the set of all Turing degrees (as described, e.g., in [9]). To verify (a)-(c), note first that

(d) (Selman [7].) For all sets $A$, if $d(A)=a$ then $a \cup 0^{\prime} \leqq d\left(H^{A}\right) \leqq a^{\prime}$. Hence $A$ is bound whenever $d\left(H^{\dot{A}}\right)=a \cup 0^{\prime}$; since the latter holds, by Fact 4, whenever $A$ is immune, it follows that

(e) Every immune set is bound.

But it is a well-known (and easily verified) fact that almost every set is immune, hence (a) holds. Also, it was proved in [1] that every nonzero degree contains an immune set; so (b) holds for nonzero degrees (for $\mathbf{0}$, it holds by Lemma 1). Finally it is clear from (d) that a degree $a$ is totally bound if $a^{\prime}=a \cup 0^{\prime}$. But the latter holds for almost all $a$, by a result attributed to Sacks in [9], which proves (c). In the present terminology, Theorems 1, 3 and 4 of the previous section give conditions under which a degree $a$ is not totally bound; in light of (a)-(c) it is clear that some strong restrictions on $a$ are in fact necessary.

\section{REFERENCES}

1. J. C. E. Dekker and J. Myhill, Retraceable sets, Canad. J. Math. 10 (1958), 357-373. MR 20 \#5733.

2. L. Hay, The class of recursively enumerable subsets of a recursively enumerable set, Pacific J. Math. 46 (1973), 167-183.

3. C. G. Jockusch, Jr., The degrees of bi-immune sets, Z. Math. Logik Grundlagen Math. 15 (1969), 135-140. MR 39 \#5360.

4. R. W. Robinson, Interpolation and embedding in the recursively enumerable degrees, Ann. of Math. (2) 93 (1971), 285-314. MR 43 \#51.

5. H. Rogers, Jr., Theory of recursive functions and effective computability, McGrawHill, New York, 1967. MR 37 \#61.

6. A. L. Selman, Applications of forcing to the degree-theory of the arithmetical hierarchy, Proc. London. Math. Soc. 25 (1972), 586-602.

7. —, Relativized halting problems (to appear).

8. J. R. Schoenfield, On degrees of unsolvability, Ann. of Math. (2) 69 (1959), 644 653. MR 21 \#4097.

9. J. Stillwell, Decidability of the "almost all" theory of degrees, J. Symbolic Logic 37 (1972), 501-506.

Department of Mathematics, University of Illinois at Chicago Circle, Chicago, Illinois 60680 